\title{
Author Correction: Classification and Visualization of Alzheimer's Disease using Volumetric Convolutional Neural Network and Transfer Learning
}

\author{
Kanghan Oh, Young-Chul Chung, Ko Woon Kim, Woo-Sung Kim \& II-Seok Oh
}

Correction to: Scientific Reports https://doi.org/10.1038/s41598-019-54548-6, published online 03 December 2019

This Article contains errors in references 5, 26, 36, 37 and 40, which were incorrectly given as:

Hayit, G., Bram van, G. \& Ronald, M. S. Guest Editorial Deep Learning in Medical Imaging: Overview and Future Promise of an Exciting New Technique. IEEE Transactions on Medical Imaging. 35, 1153-1159 (2016).

Kilian, H. et al. Multimodal Hippocampal Subfield Grading For Alzheimer's Disease Classification, https://doi. org/10.1101/293126 (2018).

Diederik, P. K. \& Jimmy, B. Adam: A Method for Stochastic Optimization. In: ICLR. 2015 (2015).

Christian, S., Vincent, V., Sergey, L. \& Zbigniew, W. Rethinking the Inception Architecture for Computer Vision. In: CVPR, https://doi.org/10.1109/CVPR.2016.308 (2016).

Loffe, S. \& Szegedy, C. Batch normalization: accelerating deep network training by reducing internal covariate shift. In ICML. 448-456 (2015).

The correct references 5, 26, 36, 37 and 40 appear below as references $1-5$, respectively.

\section{References}

1. Greenspan, H., van Ginneken, B. \& Summers, R. M. Guest Editorial Deep Learning in Medical Imaging: Overview and Future Promise of an Exciting New Technique. IEEE Transactions on Medical Imaging. 35, 1153-1159 (2016).

2. Hett, K. et al. Multimodal Hippocampal Subfield Grading For Alzheimer's Disease Classification. Sci. Rep. 9, 13845, https://doi. org/10.1038/s41598-019-49970-9 (2019).

3. Kingma, D. P. \& Ba, J. Adam: A Method for Stochastic Optimization. In: ICLR. (2015).

4. Szegedy, C., Vanhoucke, V., Ioffe, S., Shlens, J. \& Wojna, Z. Rethinking the Inception Architecture for Computer Vision. In: CVPR, https://doi.org/10.1109/CVPR.2016.308 (2016).

5. Ioffe, S. \& Szegedy, C. Batch Normalization: Accelerating Deep Network Training by Reducing Internal Covariate Shift. In: ICML. $448-456$ (2015).

(i) Open Access This article is licensed under a Creative Commons Attribution 4.0 International License, which permits use, sharing, adaptation, distribution and reproduction in any medium or format, as long as you give appropriate credit to the original author(s) and the source, provide a link to the Creative Commons license, and indicate if changes were made. The images or other third party material in this article are included in the article's Creative Commons license, unless indicated otherwise in a credit line to the material. If material is not included in the article's Creative Commons license and your intended use is not permitted by statutory regulation or exceeds the permitted use, you will need to obtain permission directly from the copyright holder. To view a copy of this license, visit http://creativecommons.org/licenses/by/4.0/.

(C) The Author(s) 2020 